\title{
GRAVIDEZ NA ADOLESCÊNCIA: MAPEAMENTO DA PRODUÇÃO CIENTÍFICA PUBLICADA EM PERIÓDICOS NACIONAIS ENTRE 2003 E 2008
}

\section{PREGNANCY IN ADOLESCENCE: MAPPING OF THE SCIENTIFIC PRODUCTION PUBLISHED IN NATIONAL JOURNALS FROM 2003 TO 2008}

\author{
Daniela Tavares Gontijo ${ }^{1}$ \\ Daniel Gustavo de Sousa Carleto \\ Sofia Martins \\ Heliana Castro Alves
}

\begin{abstract}
Resumo: A gravidez na adolescência, considerada pela Organização Mundial de Saúde, como aquela que ocorre entre os 10 e 19 anos tem sido amplamente discutida na Saúde Pública. O grande interesse social e acadêmico em relação à gravidez na adolescência no contexto brasileiro se reflete em uma numerosa produção científica sobre o tema e traz a necessidade de se realizar uma síntese desta. Assim, o objetivo desse estudo foi analisar a produção científica sobre gravidez na adolescência em periódicos nacionais da área da saúde no período de 2003 a 2008. Realizou-se uma pesquisa bibliográfica na qual os dados foram coletados na base de dados LILACS da BIREME a partir do descritor "gravidez na adolescência", e analisados a partir de estatística descritiva e análise de conteúdo. Dos 124 artigos selecionados, escritos por 426 autores, 34 foram publicados em periódicos vinculados à saúde pública. $O$ processo de categorização dos dados qualitativos resultou em 5 categorias temáticas denominadas: características das mães e pais adolescentes e seus filhos (41,1\%); Vivências da gravidez na adolescência (37,9\%); Assistência em saúde $(8,9 \%)$; sexualidade e gravidez na adolescência (9,7\%); Aborto (2,4\%). A síntese realizada neste estudo, na prática dos serviços de saúde, poderá facilitar o acesso dos profissionais a este conhecimento, o que contribuirá para o planejamento e sistematização de ações subsidiadas por evidências científicas, bem como possibilitará o aprofundamento das discussões. Além disso, o mapeamento da produção científica sobre esse tema possibilitou a identificação de lacunas em relação ao conhecimento que podem se reverter em novas demandas para a pesquisa neste campo.
\end{abstract}

Palavras chave: Gravidez. Adolescência. Juventude.

Abstract: Pregnancy in adolescence is considered by the World Health Organization the one which happens from ages 10 to 19 and has been widely discussed in public health studies. The high academic and social interest for pregnancy in adolescence in the Brazilian context is reflected by the great number of scientific studies about the theme and brings the necessity of accomplishing a synthesis of the subject. Thus, the aim of this study was to analyze the scientific production about pregnancy in adolescence in the national index of the health area in the period 2003-2008. A bibliographical survey was accomplished in which data were collected from BIREME's LILAC using "adolescent pregnancy" keyword and analyzed using descriptive statistics and content analysis. From the 124 selected articles, written by 426 authors, 34 were published in public health journals. The process of categorizing qualitative data resulted in five thematic categories, namely characteristics of teen mothers and fathers and their children (41,1\%), pregnancy experienced in adolescence (37,9\%); health assistance (8,9\%); pregnancy and sexuality in adolescence (9,7\%); abortion $(2,4 \%)$. The synthesis elaborated in this study, in health services practice, will allow access by professionals to this knowledge, contributing for the planning and systematization of

\footnotetext{
${ }^{1}$ Núcleo de Estudos e Pesquisa em Vulnerabilidade e Saúde na Infância e Adolescência- NEPVIAS Universidade Federal do Triângulo Mineiro (UFTM). Email: danielatgontijo@gmail.com.
} 
actions based by scientific evidences, as well as deepening the debate. In addition, the production mapping of the scientific production about this theme allowed the identification of lacunae related to knowledge that may create new demands regarding research in this field.

Keywords: Pregnancy. Adolescence. Youth.

\section{Introdução}

A adolescência é um período do desenvolvimento humano caracterizado por transformações biopsicossociais no qual o indivíduo vivencia novas experiências que podem deixá-lo susceptível às situações de vulnerabilidade, como uso abusivo de drogas, acidentes de trânsito, violência, contaminação por doenças sexualmente transmissíveis e gestações não planejadas (AQUINO et al., 2003).

De acordo com o Estatuto da Criança e do Adolescente (ECA) (BRASIL, 1990) na adolescência estão inseridos os indivíduos de 12 a 18 anos incompletos de idade, enquanto para a Organização Mundial de Saúde (OMS) a faixa etária varia dos 10 aos 19 anos. Entretanto, apesar da ocorrência de uma cronologia que delimita a adolescência, sabe-se que esta é influenciada por experiências individuais e pelo contexto dos sujeitos que a vivenciam (AQUINO et al., 2003; BRASIL, 2006; VIEIRA et al., 2006).

O Instituto Brasileiro de Geografia e Estatística (IBGE) revelou que em 2001 a população brasileira compunha-se de aproximadamente 32 milhões de adolescentes de 10 a 19 anos, sendo que 50,04\% desses são representados por homens e 49,96\%, por mulheres (BRASIL, 2006). Nesta faixa etária a alta prevalência da gravidez tem despertado o interesse da saúde pública, uma vez que segundo dados do DATASUS (2008), em 2005, 21,8\% de crianças nasceram de mães adolescentes.

De uma forma geral, os autores discutem que a ocorrência da gravidez na adolescência pode ser influenciada por inúmeros fatores, desde aqueles com origem social aos de caráter biológico, tais como contexto econômico desfavorecido; desejo de inserção precoce na vida adulta; mudança de status e obtenção de prestígio social; repetição de história familiar; desconhecimento e uso inadequado de métodos contraceptivos; relações de gênero; diminuição da idade de menarca; carência de serviços específicos para atender essa faixa etária; e outros fatores ligados à 
subjetividade do adolescente (GONTIJO; MEDEIROS, 2004; MONTEIRO et al., 2007). Por outro lado, os estudos que discutem as conseqüências da gravidez na trajetória de vida das adolescentes, trazem que desorganização familiar, estabelecimento de união conjugal, tentativa de aborto, abandono do parceiro, não aceitação da família e discriminação social são situações freqüentes no cotidiano de adolescentes que engravidam, assim como a evasão escolar, dificuldade para inserção no mundo do trabalho e o isolamento social (SANTOS; SCHOR, 2003; DIAS; AQUINO, 2006).

Entretanto, apesar do caráter vitimizador, homogeneizador e alarmista dado a esse tema, ao qual se associam características negativas e transformações radicais, quando a gravidez nessa faixa etária é analisada sob a perspectiva dos sujeitos que a vivenciam, aspectos como concretização de sonhos, realização de projetos pessoais e alternativas de vida podem ser percebidos como situações relevantes relacionadas a esta experiência (HEILBORN et al., 2002; GONTIJO; MEDEIROS, 2008).

O grande interesse social e acadêmico em relação à gravidez na adolescência no contexto brasileiro se reflete em uma numerosa produção científica sobre o tema e traz a necessidade de se realizar uma síntese desta. Esta síntese, na prática dos serviços de saúde, pode facilitar o acesso dos profissionais a este conhecimento, o que contribui para o planejamento e sistematização de ações subsidiadas por evidências científicas, bem como possibilita o aprofundamento das discussões. Além disso, ao se mapear a produção científica sobre um determinado tema, torna-se possível identificação de lacunas em relação ao conhecimento que podem se reverter em novas demandas para a pesquisa neste campo.

Assim, o objetivo desse estudo é analisar a produção científica sobre gravidez na adolescência em periódicos nacionais da área da saúde no período de 2003 a 2008.

\section{Percurso Metodológico}

De acordo com Gomes e Nascimento (2006) há inúmeros caminhos para se refletir sobre a produção do conhecimento acerca de um determinado tema. Nesse estudo optou-se por uma pesquisa bibliográfica do tipo revisão crítica, na qual se 
objetiva resumir, analisar e sintetizar as informações disponíveis na literatura (MANCINI; SAMPAIO, 2006).

Os dados foram coletados na Biblioteca Virtual em Saúde (BIREME), que tem o objetivo de promover, aprimorar e disseminar o conhecimento técnicocientífico, contribuindo para o desenvolvimento da saúde nos países da América Latina e do Caribe (GOLDBAUM, 2000). A base de dados utilizada nessa pesquisa foi a Literatura Latino-Americana e do Caribe em Ciências da Saúde (LILACS), que, segundo Benetti et al (2007, p.1274), inclui "informações e referências sobre a literatura internacional e científico-técnica da América Latina e do Caribe". Esta escolha se justifica pela constatação da indexação de grande número de periódicos nacionais nesta base de dados.

Foram incluídos no estudo os resumos de artigos científicos que utilizaram gravidez na adolescência como Descritor em Ciência da Saúde (DECS), publicados em periódicos brasileiros entre 2003 e 2008. Foram excluídos os resultados que se caracterizaram como outros tipos de publicações (teses, capítulos de livros e livros), artigos que não possuíam resumo disponível e aqueles cujo foco principal não era compatível com o assunto da pesquisa.

Inicialmente os dados foram sistematizados em um protocolo elaborado pelos pesquisadores, no qual foram alocadas as seguintes variáveis: autores, revista, área científica da revista, ano, tipo de pesquisa, abordagem metodológica, sujeitos, título, objetivos e resultados de estudo.

As variáveis: título, objetivo e resultados foram submetidas à análise de conteúdo proposta por Bardin (1979), e o processo resultou em cinco categorias de análise: Características das(dos) Mães/Pais Adolescentes e seus filhos; Vivências da Gravidez na Adolescência; Assistência em saúde; Sexualidade e Gravidez na Adolescência e Aborto. As outras variáveis foram submetidas à análise estatística descritiva, através da qual foi possível a construção de indicadores de freqüência absoluta e relativa. 


\section{Resultados e Discussão}

$\mathrm{Na}$ primeira etapa da pesquisa, a consulta à base de dados indicou 418 artigos científicos publicados no período de 2003 a 2008, de acordo com o Descritor em Ciência da Saúde (DeCS) "gravidez na adolescência”. Destes, 295 foram excluídos, pois se caracterizavam como teses, capítulos de livros, livros, artigos que não possuíam resumo disponível e aqueles cujo foco principal de discussão não correspondia à gravidez na adolescência.

Em relação ao número de artigos encontrados a partir do descritor, é importante pontuar que o número total de artigos pode não corresponder a toda a produção sobre o tema na referida base de dados, uma vez que existe a possibilidade de alguns pesquisadores não terem utilizado o descritor "gravidez na adolescência" como palavra chave de suas pesquisas.

A partir dos critérios de inclusão e exclusão da pesquisa, 124 artigos foram selecionados $^{2}$, sendo 19,3\% publicados em 2003, 15,32\% em 2004, 20, $2 \%$ em 2005, 20,2\% em 2006, 15,3\% em 2007 e 9,7\% em 2008.

Em relação ao número de autores que escreveram sobre o tema, foram identificados 368 pesquisadores, refletindo o interesse de um grande número de pesquisadores sobre o tema e consequentemente a possibilidade da co-existência de diferentes perspectivas e análises em relação ao fenômeno em estudo, que se refletem nas categorias temáticas discutidas posteriormente.

Os artigos foram publicados em 53 periódicos (tabela 1), sendo que quando se analisa as áreas científicas de todos os periódicos nos quais os 124 artigos foram publicados, observa-se uma maior concentração de publicações no campo da saúde pública $(32,1 \%)$ e da enfermagem $(26,8 \%)$.

\footnotetext{
${ }^{2} \mathrm{~A}$ listagem completa dos resumos selecionados encontra-se ao final do artigo.
} 
Tabela 1. Lista de Periódicos com publicações de três ou mais artigos sobre gravidez na adolescência no período de 2003 a 2008.

\begin{tabular}{lc}
\hline Periódico & $\begin{array}{c}\text { Número de artigos } \\
\text { publicados }\end{array}$ \\
\hline Cadernos de Saúde Pública & 20 \\
Revista LatinoAmericana de Enfermagem & 9 \\
Revista de Saúde Pública & 8 \\
Escola Anna Nery - Revista de Enfermagem & 7 \\
Ciência e Saúde coletiva & 5 \\
Revista Brasileira de Saúde Materno Infantil & 5 \\
Revista de Enfermagem da UERJ & 5 \\
Revista Brasileira de Crescimento e Desenvolvimento & 4 \\
Humano & \\
Pediatria (São Paulo) & 3 \\
Revista Brasileira Enfermagem & 3 \\
Revista Brasileira Ginecologia e Obstetrícia & 3 \\
Acta Paulista de Enfermagem & 3 \\
Outras Revistas (número de artigos inferior a 3) & 49 \\
\hline Total & \\
\hline
\end{tabular}

A concentração de publicações no campo da saúde pública confirma a representação social da gravidez na adolescência como um "problema de saúde pública", sendo pertinente então a sua caracterização e discussão por esse campo do conhecimento. Por outro lado, o interesse no tema no campo da enfermagem pode estar relacionado com a intervenção tradicional destes profissionais no campo da obstetrícia e pediatria, uma vez que estes proporcionam estratégias de cuidados tanto para a adolescente, como para seu filho e família, além de intervenções direcionadas para a educação em saúde reprodutiva (ALMEIDA et al., 2003; FOLLE; GEIB, 2004).

Em relação às opções metodológicas utilizadas pelos autores para abordarem o objeto de estudo, percebeu-se que $73,3 \%$ das pesquisas se caracterizam como pesquisas de campo, 12,9\% como pesquisas documentais, 8,8\% como pesquisas bibliográficas e em $4,8 \%$ dos estudos não foi possível caracterizar o tipo de pesquisa a partir da leitura do resumo. O grande percentual de pesquisas direcionadas pelo trabalho de campo reflete um maior interesse dos pesquisadores em torno da caracterização do fenômeno na sociedade brasileira, seja em busca de indicadores quantitativos necessários para o planejamento e avaliação de ações de 
saúde ou da compreensão do impacto desta experiência e das intervenções realizadas na vida dos e das adolescentes.

Especificamente em relação às pesquisas de campo, 59,5\% utilizaram métodos quantitativos para abordarem o método de estudo, 29,2\% o qualitativo, 2,3\% o qualitativo-quantitativo e em $8,69 \%$ dos resumos não foi possível identificar o tipo de abordagem utilizada.

A predileção dos pesquisadores por métodos quantitativos pode ser discutida com base em dois aspectos. Inicialmente, é importante considerar que a maioria dos estudos se insere no contexto da saúde pública, onde a utilização de indicadores quantitativos, que possibilitam a generalização dos resultados para grupos mais amplos da população, são de vital importância para o planejamento, sistematização e avaliação das intervenções realizadas. No entanto, apesar de ainda incipiente, observa-se 0 interesse crescente de pesquisadores em obter informações de cunho qualitativo que possibilitam o aprofundamento das questões, bem como o direcionamento das ações com base na realidade sócio-histórica e cultural de populações alvo especificas. Um outro aspecto que pode ser relacionado à predileção por métodos quantitativos em detrimento de métodos qualitativos refere-se às concepções hegemônicas acerca do que é cientifico ou não, sendo enfatizada a busca pela regularidade e normatividade dos fatos, características estas pertinentes ao campo dos métodos quantitativos. No entanto, consoantes a Sanches e Minayo (1993), acreditamos que a discussão em torno da escolha de métodos quantitativos ou qualitativos deve estar relacionada à concepção de complementaridade destes, sendo a natureza do objeto de pesquisa o fator determinante da escolha do método a ser utilizado.

Ainda em relação às pesquisas de campo, quando analisamos os sujeitos que participaram dos estudos, duas porcentagens se destacam: 67,6\% são adolescentes do sexo feminino que vivenciaram ou não a maternidade e/ou gravidez na adolescência e 5,6\% correspondem aos pais ou adolescentes do sexo masculino. Apenas um dos estudos utilizou os dois gêneros numa pesquisa de campo que abordou a sexualidade na adolescência (SOARES et. al, 2008). Essa significativa diferença em relação aos participantes do estudo reflete as concepções hegemônicas de gênero acerca da reprodução e cuidado de crianças. Tradicionalmente o controle da reprodução bem como a gravidez e cuidado das 
crianças se caracterizam como tarefas destinadas às mulheres, sendo observado um distanciamento dos homens nestes assuntos. No entanto, tanto no campo social de uma forma geral, como das políticas públicas tem-se debatido a necessidade de desenvolvimento de estratégias que busquem possibilitar e incentivar uma maior participação dos homens no campo da reprodução e cuidados de crianças (BRASIL, 2008).

A partir da análise dos dados qualitativos referentes aos objetivos dos estudos e resultados apresentados nos resumos, emergiram cinco categorias de análise.

De uma forma geral, como explícito na Tabela 2, foi possível observar um maior interesse dos pesquisadores em descrever o perfil das pessoas que vivenciam a gravidez na adolescência, assim como na discussão sobre as diferentes vivências e repercussões desta experiência. Além disso, observa-se um interesse incipiente na pesquisa sobre a assistência em saúde nesta fase da vida. Por outro lado, são poucos os estudos que discutem a questão do aborto, tema tradicionalmente interdito em nossa sociedade, de forma relacionada à gravidez na adolescência.

Tabela 2. Número de artigos por categoria temática.

\begin{tabular}{lcc}
\hline Categoria & $\begin{array}{c}\text { Número de artigos } \\
\text { publicados }(\mathrm{n}=124)\end{array}$ & $\%$ \\
\hline $\begin{array}{l}\text { Características das mães e pais } \\
\text { adolescentes e seus filhos }\end{array}$ & 51 & 41,1 \\
Vivencias da gravidez e maternidade na & 47 & 37,9 \\
adolescência & & \\
Assistência em saúde & 11 & 8,9 \\
Sexualidade e gravidez na adolescência & 12 & 9,7 \\
Aborto & 3 & 2,4 \\
\hline
\end{tabular}

$\mathrm{Na} 1^{1}$ a categoria de análise, Características de mães e pais adolescentes e seus filhos, que corresponde a $41 \%$ dos artigos publicados, foram incluídos os estudos que abordavam o perfil de jovens mães e pais adolescentes, principalmente no que se refere aos aspectos sócio-demográficos e à prevalência e recorrência da gravidez, condições de saúde e doenças associadas à gravidez na adolescência, bem como características do recém-nascido.

Nesta categoria, os estudos, em sua maioria de natureza descritiva, trazem o perfil das adolescentes mães que freqüentam serviços públicos de acompanhamento em saúde (PINHO, MEINCKE, 2003; DEL CIAMPO et al., 2003; 
MAIA, ANDRADE; MAAKAROUN, 2003; SABROZA et al., 2004; PAIVA et al., 2004; PERSONA; SHIMO; TARALLO, 2004; MAUCH et al., 2005; PARAGUASSÚ et al., 2005; BORGES; SCHOR, 2005; SANTOS; CARVALHO, 2006; TAVARES, FERRARI; SOLER, 2006; CARNIEL et al., 2006; KASSAR et al., 2006; ARCANJO, OLIVEIRA; BEZERRA, 2007; BARALDI et al., 2007 ; CHALEM et al., 2007) .É importante pontuar que poucos estudos buscaram caracterizar este perfil em outros contextos, como serviços particulares de saúde (BARALDI et al., 2007) e na população em geral (AQUINO et al., 2003 ; DIAS; AQUINO, 2006; GONÇALVES; GIGANTE, 2006; BRANDÃO; HEILBORN, 2006).

De uma forma geral, os estudos caracterizam como precoce o início da atividade sexual e da ocorrência da gravidez para estas adolescentes (PAIVA et al., 2004; DIAS; AQUINO, 2006; TAVARES; FERRARI; SOLER, 2006; KASSAR et al., 2006; ARCANJO; OLIVEIRA; BEZERRA, 2007; BERALDI et al., 2007).

Neste aspecto, é importante pontuarmos que a visão de precocidade da iniciação sexual bem como da concepção da gravidez enquanto precoce nesta fase da vida, precisa ser discutida de forma contextualizada histórica e socialmente, articulada às demandas sociais direcionadas para determinado grupo social e ainda às significações subjetivas deste grupo populacional em relação à vivência da gravidez, considerando sua inserção na cultura.

Nota-se, neste sentido, as contribuições de outros campos do conhecimento sobre os significados sociais em torno de alguns fenômenos como a gravidez na adolescência também em diferentes contextos sócio-hitóricos. Heilborn (1998), a partir do olhar da antropologia, realiza uma ressalva quanto ao modo como a gravidez na adolescência vem sendo construída, mostrando uma mudança na percepção social acerca do tempo certo para uma gestação. Assim, as expectativas sociais em torno da idade para gerar descendentes são alteradas sócio e historicamente, ou seja, o que em dado momento é tido como natural, em outro contexto, se considera inaceitável.

Como discutido por Gontijo e Medeiros (2004) o fenômeno da gravidez na adolescência, que não se caracteriza como um fato recente no Brasil ganha notoriedade acadêmica e social a partir da década de 60 , data em que se observa um contexto histórico de discussões e mudanças nos valores referentes aos conceitos de gênero e sexo, demarcados principalmente por uma valorização 
crescente da dissociação entre atividade sexual e reprodutiva, da busca por maior escolaridade e inserção da mulher no mundo do trabalho.

A partir deste momento, a gravidez na adolescência passa a ser considerada "um problema social", sustentada por um discurso médico que a caracterizou como quadro de gravidade e risco (GONTIJO; MEDEIROS, 2004). No entanto, conforme pontua Heilborn et al. (2002) e Gontijo e Medeiros (2008) é importante considerar que as oportunidades de maior escolarização e inserção no mundo do trabalho não são igualmente oferecidas a todos os jovens de todas as classes sociais. Neste sentido, conforme discutido posteriormente na categoria referente às vivências da gravidez, em contextos com poucas oportunidades de ascensão social, marcados por privações de ordem econômica e afetiva, a gravidez pode se caracterizar enquanto um projeto de vida, e sendo assim, como uma experiência desejada e esperada por muitas adolescentes.

No entanto, quando se analisa os estudos que buscam a caracterização do perfil da e do adolescente que vivencia a gravidez, percebe-se uma concepção fortemente marcada pela visão do risco permeando parte destes estudos que compuseram esta categoria, uma vez que procuram caracterizar o "problema" a partir dos fatores de risco a ele relacionados (CHALEM et al., 2007; MAUCH et al., 2005 ; CARNIEL et al., 2006; SILVA; INAGAKI; DALTRO, 2008).

Esta visão, sustentada muitas vezes, por estudos de natureza somente descritiva, é contraditória aos estudos que comparam diferentes populações de adolescentes. Estes estudos (KASSAR et al., 2006; PINTO et al., 2005; KASSAR et al., 2005; BORGES; SCHOR, 2005; COSTA-PAIVA et al., 2004), de uma maneira geral, apontam que os riscos tanto para a adolescente, quanto para o bebê, se referem muitos mais às condições de precariedade socioeconômicas vivenciadas, do que à gravidez em si, ou seja, às características maturacionais da adolescente (exceto no caso de meninas abaixo de 14 anos).

As relações entre gravidez na adolescência e pobreza são amplamente discutidas na literatura, conforme pontua Gontijo e Medeiros (2004). No entanto, os autores, cujos estudos compuseram esta pesquisa, apontam para a necessidade de não se compreender o fenômeno de forma simplista, buscando o estabelecimento de relações causais diretas para uma realidade que é marcada pela complexidade (AQUINO et al., 2003; DEL CIAMPO et al., 2003; NUNES et al., 2005; OTSUKA et 
al., 2005; BRANDÃO; HEILBORN, 2006; BORGES; SCHOR, 2005; CAPUTO; BORDIN, 2007). Neste sentido, torna-se necessário, para o planejamento de ações de prevenção à gravidez indesejada não só a utilização de indicadores numéricos sobre a sua ocorrência, mas também a adoção de indicadores qualitativos que dizem respeito aos processos de significação destas experiências, conforme discutido na próxima categoria Vivências da Gravidez e Maternidade na Adolescência.

Ainda em relação às características das mães e pais adolescentes e seus filhos, a complexidade do fenômeno em estudo também se reflete nos estudos que buscaram caracterizar a recorrência da gravidez na adolescência (PERSONA; SHIMO; TARALLO, 2004; BERLOFI et al., 2006; CUNHA; BRUNO, 2007; ROSA; REIS; TANAKA, 2007). De uma forma geral, discute-se que a redução da reincidência não depende somente de programas de planejamento familiar que visam o uso adequado de métodos contraceptivos, uma vez que outros fatores como escolaridade das adolescentes, retorno à escola e estabelecimento de união conjugal após a primeira gestação também contribuem para que isso ocorra. Acredita-se que, assim como uma primeira gravidez, para muitos adolescentes a recorrência do fenômeno pode estar relacionada a uma mudança de status social, principalmente quando estes passam a constituir uma família. Portanto, sugere-se que programas de planejamento familiar tenham o objetivo de oferecer opções de vida para os adolescentes, incluindo, entre essas opções, o desejo de ter mais filhos.

Ao realizar uma caracterização deste público em relação às condições de saúde e doenças associadas à gravidez no período da adolescência, os artigos discutem o impacto de diferentes condições para a adolescente e seu filho. Neste sentido, são discutidos aspectos relacionados ao estado nutricional da adolescente (PAPA et al., 2003; NOGUEIRA; PARENTE; COZZOLINO, 2003; BARROS et al., 2004; GUERRA; HEYDE; MULINARI, 2007) , tabagismo (VITALLE; AMANCIO, 2003), obesidade (KAC; VELÁSQUEZ-MELÉNDEZ; VALENTE, 2003), HIV (PAIVA; GALVÃO, 2006), febre reumática (MORAES et al., 2004), dermatomiosite (SILVA et al., 2005) , problemas psiquiátricos (NUNES et al. , 2005; CAPUTO; BORDIN, 2007) e uso de drogas durante a gravidez (MITSUHIRO et al., 2006). 
Um último aspecto que chama a atenção não por sua presença, mas por sua ausência na caracterização do perfil referente à gravidez na adolescência, refere-se ao número ínfimo de trabalhos que discutem o perfil do adolescente do sexo masculino que vivencia a gravidez na adolescência (DIAS; AQUINO; ESTELA, 2006; AQUINO et al., 2003), caracterizando o fenômeno denominado por Lyra (1998) como de invisibilidade da paternidade adolescente. Conforme pontuado anteriormente, este fato está relacionado as concepções hegemônicas de gênero, nas quais o cuidado dos filhos, bem como o controle da vida sexual e reprodutiva são tarefas de domínio feminino (Brasil, 2006).

$\mathrm{Na} 2^{a}$ categoria de análise, Vivências da Gravidez e Maternidade na Adolescência, foram alocados $37,9 \%$ dos estudos que compuseram esta pesquisa. De uma forma geral, a maioria dos estudos buscou analisar os significados atribuídos pela mãe adolescente à experiência da gravidez e maternidade, bem como os impactos na sua trajetória de vida. Os estudos foram realizados em sua totalidade, com adolescentes em situações de vulnerabilidade social, e apontam as contradições entre a concepção da gravidez na adolescência como situação que resulta em abandono escolar e ruptura dos projetos de vida, e as percepções que a compreendem como estratégia de poder das adolescentes para uma possível e futura mudança de status social (ANDRADE; RIBEIRO; SILVA, 2003; ALMEIDA et al., 2003; PANTOJA, 2003; SANTOS; SCHOR, 2003; MENEZES; DOMINGUES, 2004; LEAL; WALL, 2005; MORESCO, 2005;REIS, CAVALCANTI, 2005; SANTOS; CARVALHO, 2006; JORGE; FIUZA; QUEIROZ, 2006; HOGA , 2008; GONTIJO E MEDEIROS, 2008).

Nesta categoria alguns artigos enfocam as características das relações estabelecidas pela adolescente com o bebê, apontando uma ausência de consenso entre estes, pois alguns apontam diferenças nas relações em virtude da idade da mãe, e outros discutem que estas diferenças podem estar relacionadas a uma multiplicidade de fatores. (EDUARDO, et al., 2005; ROCHA; BEZERRA; CAMPOS, 2005; FOLLE; GEIB, 2004; PICCININI et al., 2003; MARQUES et al., 2008; PEDRO et al., 2007; BIGRAS; PAQUETTE, 2007).

Diversos estudos abordaram a importância do suporte familiar e sua influência nos processos de significação da gravidez na adolescência (MOREIRA et al., 2008; SABROZA et al., 2004; FERNANDES; SOUSA; BARROSO, 2004; MOTTA 
et al., 2004; FALCAO; SALOMÃO, 2005; MONTES et al., 2006; MONTEIRO et al., 2007; SILVA; TONETE, 2006; PARAGUASSU et al., 2005). De forma unânime, os pesquisadores apontam a importância do suporte familiar para a vivência da gravidez na adolescência de uma forma positiva, e destacam a necessidade dos profissionais de saúde também elaborarem estratégias de ação direcionadas para a valorização e apoio das famílias.

Embora a gravidez na adolescência seja tradicionalmente associada a uma concepção de risco biopsicossocial, tanto para a mãe como para a criança, resultados de estudos com abordagem qualitativa sobre o tema sugerem que os sentidos desta experiência não são únicos ou definitivos, podendo apresentar aspectos positivos e negativos para os adolescentes (CABRAL, 2003; SANTOS; SCHOR, 2003).

Assim, a partir da análise dos resumos foi possível constatar que a vivência da gravidez na adolescência não é significada de forma homogênea, devendo ser compreendida como um fenômeno complexo que requer ações assistenciais sustentadas pela integralidade e por ações intersetoriais. A necessidade da articulação de ações intersetoriais se justifica pela percepção das relações entre gravidez na adolescência e condições de vida. Neste sentido, espera-se que a promoção de uma maior equidade no acesso aos bens sociais (educação, trabalho, moradia, lazer, etc) possa resultar em um maior empoderamento das adolescentes em seus contextos reais de vida, que se refletirá (refletindo) em maiores oportunidades de escolhas para estas no que se refere à sua saúde sexual e reprodutiva.

$\mathrm{Na} 3^{\text {a }}$ categoria de análise, Assistência em Saúde, que corresponde a somente $8,9 \%$ dos artigos publicados, foram incluídos os resumos especificamente a assistência em saúde no que se refere à gravidez na adolescência. Maturana e Progianti (2007) e Gurgel et al (2008) discutem especificamente os aspectos relacionados à assistência em enfermagem, apontando a necessidade de 0 profissional contextualizar a sua prática clínica com o contexto no qual ocorre. Além disso, são relatadas algumas experiências assistenciais, principalmente de caráter educativo e grupal, nas quais os autores reforçam a necessidade do oferecimento de programas de promoção de saúde direcionados para este público e a necessidade de capacitação profissional. 
Nesta categoria, dois estudos se destacam por trazerem dados interessantes e passíveis de maiores explorações pelos pesquisadores. Fonseca (2003) buscou compreender como os profissionais percebem a adolescente gestante, encontrando percepções contraditórias: ora a gestante é aceita com naturalidade pela equipe de saúde e ora é compreendida como cliente de risco e, Daltoso (2005) apresentou a avaliação que um grupo de adolescente realizou referente à assistência recebida em uma maternidade. Estes dois estudos trazem à tona a percepção de diferentes atores envolvidos na assistência, cuja maior "escuta" por parte dos responsáveis pela elaboração desta, pode resultar em uma maior resolutividade das ações.

$\mathrm{Na} 4^{\circ}$ categoria de análise, Sexualidade e Gravidez na Adolescência, que corresponde a $9,7 \%$ dos artigos publicados, os resumos discorreram sobre conhecimentos dos e das jovens sobre práticas sexuais, experiências sexuais, informações sobre sexualidade, intenção reprodutiva e métodos contraceptivos.

Nessa categoria, o assunto mais abordado foi sobre métodos contraceptivos. De uma forma geral discute-se que apesar de os adolescentes terem conhecimento sobre os métodos contraceptivos e concordarem com sua utilização, não recebem orientações corretas sobre seu uso (MAIA; ANDRADE; MAAKAROUN, 2003; BELO; SILVA, 2004; VIEIRA et al., 2006).

Ao se analisar as informações sobre sexualidade que os adolescentes recebem, Romero et al. (2007) discutem que a maioria busca essas informações e que o conhecimento que têm sobre doenças sexualmente transmissíveis e contracepção ainda são inadequados. Além disso, Borges, Nichiata e Schor (2006) sugerem que há necessidade de incluir os sujeitos que dialogam sobre sexo com os adolescentes nas ações de promoção à saúde sexual e reprodutiva dos mesmos.

Em relação às experiências sexuais dos adolescentes, discute-se que abordagens sobre sexo seguro que priorizam os diferentes contextos sócio-culturais dos adolescentes são necessárias e o que o namoro e as questões familiares são aspectos que necessitam ser incorporados na formulação de políticas de saúde reprodutiva e sexual voltadas aos adolescentes (VILLELA; DORETO, 2006; BORGES; LATORRE; SCHOR, 2007).

É importante refletir sobre o pequeno número de estudos relacionados à sexualidade e gravidez na adolescência. Neste sentido, acredita-se que este número 
não abarca o contingente total de estudos, uma vez que existe a possibilidade dos autores que trabalharam com temas relacionados à sexualidade na adolescência não terem utilizado o descritor "gravidez na adolescência" em seus artigos. A nãoutilização destes descritores também pode significar que os assuntos abordados sobre sexualidade pelos pesquisadores não se restringem à discussão da gravidez na adolescência ao mesmo tempo em que a abarca entre outros assuntos relacionados. Isso, provavelmente, reflete na utilização de outros descritores.

$\mathrm{Na} 5^{\text {a }}$ categoria de análise, Aborto, foram alocados 3 artigos (2,4\%) que direcionam a sua atenção para a descrição e discussão do aborto de forma relacionada à gravidez na adolescência, sendo que os autores apontam a necessidade de implantação de políticas e programas direcionados à saúde sexual dos adolescentes, contribuindo para a realização segura e saudável de seus projetos reprodutivos (MENEZES; AQUINO; SILVA, 2006; PERES; HEILBORN, 2006; VIEIRA et al., 2007).

\section{Considerações finais}

A gravidez na adolescência, enquanto um fenômeno que ganhou destaque na saúde pública, deve ser compreendida de forma ampla e numa perspectiva sóciohistórica reconhecendo o processo de mudança que influencia as representações sociais e a produção de conhecimento.

Um primeiro aspecto que devemos refletir se refere à possibilidade de que muitos estudos sobre o tema não terem sido incluídos nesta pesquisa em virtude da não utilização do descritor gravidez na adolescência como palavra chave pelos autores. Por outro lado, na busca inicial foram identificados muitos artigos que utilizaram o descritor em questão, sendo que, no entanto, a partir da leitura do resumo, foi possível identificar que a gravidez na adolescência não era o foco do estudo. Estes achados apontam para a necessidade de um cuidado criterioso dos autores na seleção dos descritores utilizados em seus artigos de forma a facilitar o acesso à produção sobre determinado tema. Da mesma forma, é importante pontuar que a revisão apresentada pode não representar toda a produção científica nacional, uma vez que existe a possibilidade de muitos artigos estarem publicados em periódicos internacionais ou em outras bases de dados da saúde e educação. 
Além disso, foram lidos apenas os resumos e não os artigos completos, o que pode inviabilizar o conhecimento do conteúdo de alguns artigos possivelmente relacionados que continham poucas informações no resumo acessado. Acredita-se, porém que, de acordo com o objetivo proposto, a estratégia de leitura restrita aos resumos dos artigos é assertiva, uma vez que a intenção foi a de vislumbrar um cenário geral da produção do conhecimento, e não detalhá-lo em suas especificidades.

A idéia inicial dessa pesquisa era a de realizar uma análise minuciosa de tendências, porém, um número significativo de resumos estavam incompletos, sem detalhes importantes para o acesso de informações relevantes para sua análise, como, por exemplo, metodologia, abordagem, análise e tratamento de dados, limitando a síntese da produção de conhecimento sobre o tema em questão. Neste sentido, é importante enfatizar que a questionável qualidade na constituição dos resumos analisados, pode impossibilitar o acesso de pesquisadores aos artigos e prejudicar possíveis pesquisas sobre o tema.

Em relação à inserção do tema no campo da saúde pública, é importante sinalizar que as novas perspectivas apontadas pela concepção de promoção de saúde inscrita nos diversos movimentos internacionais e nacionais neste campo, desde a publicação do informe Lalonde e a Carta de Otawa, trazem conceitos fundamentados numa definição mais ampla de saúde, redimensionando as práticas neste setor que deixam de se centrar num modelo médico-assistencial privatista pautado na concepção de saúde como ausência de doenças. Neste conceito ampliado de saúde, também se contempla a expressão de formas de organização da vida social, a paz, a educação, a moradia, a alimentação, a renda, um ecossistema saudável, justiça social e a equidade (BUSS, 2003). Segundo o autor, a Carta de Otawa enfatiza que as ações comunitárias serão efetivas se for garantida a participação popular na direção dos assuntos de saúde- o conceito de empowerment comunitário.

Considera-se ainda a importância do surgimento de práticas que busquem a inserção dos profissionais de saúde no contexto social a partir da perspectiva da saúde coletiva. Para tanto, chama-se a atenção para a necessidade de capacitação profissional que qualifique agentes de saúde na gestão de ações educativas que levem em consideração o contexto sócio-histórico e os processos de significação 
dos sujeitos-alvo inseridos em determinadas comunidades, possibilitando, desta forma, o efetivo empoderamento da população assistida, não só na formulação de políticas públicas, mas na gestão de práticas voltadas para suas necessidades e contextos de vida.

Ao considerar a perspectiva da Organização Mundial da Saúde sobre a qualidade de vida, contempla-se a posição do indivíduo sobre sua vida, no contexto da cultura e do sistema de valores que experiência e em relação ao seus objetivos, expectativas, padrões e preocupações. Segundo Gonçalves e Vilarta (apud GONÇALVES et al., 2006), registram-se duas concepções relevantes para compreender a qualidade de vida: uma, referente a dimensões emocionais, culturais e sociais e outra, objetiva, acerca das condições materiais, posição na vida da pessoa e relações estabelecidas na sociedade.

Neste sentido, a diferença de apenas três pontos percentuais entre pesquisas voltadas para a caracterização do perfil de adolescentes e as vivências da gravidez demonstra um interesse crescente nos processos de significação por parte dos atores que vivenciam a gravidez e maternidade na adolescência.

Os resultados apontam, portanto para uma necessidade de estudos que tratem mais amplamente dos significados da gravidez e maternidade na adolescência e sob diferentes perspectivas, contemplando a dimensão sócio-cultural do/ da adolescente na situação da gravidez, e sua percepção de saúde e condições para a maternidade. Percebe-se, por exemplo, a falta de estudos que se proponham a realizar reflexões sobre as experiências entre adolescentes de diferentes estratos sociais. Estudos desta natureza, com um enfoque sobre significados intrínsecos à vivência de acordo com cada contexto experienciado pelo/ pela adolescente, possibilitaria verificar se os "riscos" apontados pelos estudos quantitativos, estão relacionados à adolescência em si, enquanto período de vida peculiar que encerra fatores de vulnerabilização, ou se advém, realmente da condição sócio-econômica da parturiente.

Desta forma, percebe-se que a produção de conhecimento nos últimos anos tem se direcionado mais para a explicação e caracterização do perfil dos adolescentes em situação de gravidez (51\%), do que realmente para a caracterização da assistência em saúde (11\%). Nota-se também, neste sentido, uma necessidade de estudos que realizem uma avaliação dos programas de assistência 
a este público-alvo, para além da caracterização simples dos atendimentos prestados á população em questão na forma de relatos de experiência. A lacuna por estudos de cunho quali-quantitativo de avaliação de programas de assistência para os adolescentes que vivenciam a gravidez, pode evidenciar práticas em construção que requerem sistematização de métodos avaliativos objetivando mensurar e qualificar a efetividade das ações direcionadas. Esta perspectiva na produção de conhecimento sobre a temática possibilitaria, por sua vez, uma construção sólida acerca das formas de enfrentamento aos possíveis riscos relacionados à gravidez, bem como a garantia da qualidade de vida dos adolescentes em vista dos processos de significação desta vivência.

A paternidade na adolescência é um outro assunto pouco estudado nas pesquisas analisadas, denotando uma percepção social em relação à gravidez ainda focada no papel da mulher para a manutenção dos filhos. Desta forma, os resultados encontrados apontam para uma relevante manifestação de gênero a partir do momento que a gravidez na adolescência é caracterizada como um fenômeno analisado sob a perspectiva feminina sem considerar a representação social dos adolescentes do sexo masculino em relação à vivência deste fenômeno. No entanto, a paternidade na adolescência também deve ser vista sob um ângulo sócio-histórico e compreendida, sobretudo, na perspectiva de seus atores a partir de relatos que mostrem o impacto da gravidez sobre sua vida.

A pesquisa quali-quanti permite a complementariedade dos dados, favorecendo a coleta de dados de naturezas distintas, numéricas $\backslash$ objetivas para caracterização do contexto, e subjetivas, a partir da significação e concepção dos sujeitos que participam dos respectivos estudos. Assim, a pesquisa qualitativa pode apoiar a pesquisa quantitativa e vice-versa, sendo que ambas combinadas possibilitam a oferta de um quadro mais geral do tema pesquisado (FLICK, 2009).

A importância de estudos de revisão bibliográfica está justamente na possibilidade de se mapear o cenário da produção de conhecimento nas suas diversas áreas, potencializando a atividade de pesquisa a partir das lacunas apontadas e da análise sobre os estudos realizados. Além disso, um estudo de revisão também possibilita um acesso mais amplo de informações para os profissionais que trabalham com a temática, indicando caminhos para a prática profissional pautada em evidências. No entanto, a análise de pesquisas realizadas 
num certo período requer, antes de tudo, que se compreenda a historicidade do saber que é produzido, possibilitando, assim uma apreensão mais precisa e ampla do objeto de estudo em questão.

\section{REFERÊNCIAS}

BUSS, P. M. Uma introdução ao conceito de promoção de saúde. In: CZERESNIA, D; FREITAS, C.M (org). Promoção da Saúde: conceitos, reflexões, tendências. Editora Fiocruz: Riode Janeiro. Cap.1. p.15-37, 2003.

FLICK, U. Introdução à pesquisa qualitativa. 3ª ed. Porto Alegre: ArtMed, 2009.

GONÇALVES et cols, 2006. Saúde coletiva e atividade física: recortes atuais de sua atuação. Conexões. Revista da Faculdade de Educação Física da Unicamp, Campinas, v. 4, n. 1, 2006

HEILBORN, M. L (et. Cols). Aproximações socioantropológicas sobre a gravidez na adolescência. Ver. Horizontes antropológicos. Porto Alegre, ano 2 n. 17. P. 13 a 45. Junho de 2002.

BRASIL. Lei n. 8.069, de 13 de julho de 1990. Dispõe sobre o Estatuto da Criança e do Adolescente. Brasília: Secretaria de Estado dos Direitos Humanos, Departamento da Criança e do Adolescente, 2004.

BARDIN, L. Análise de Conteúdo. Edições 70: Lisboa, 1979.

BENETTI, S.P.C. et al. Adolescência e saúde mental: revisão de artigos brasileiros publicados em periódicos nacionais. Cad. Saúde Pública, Rio de Janeiro, 23 (6): 1273-1282, jun, 2007.

BRASIL, MINISTÉRIO DA SAÚDE. Marco teórico e referencial: saúde reprodutiva e sexual de adolescentes e jovens. Brasília: Ministério da Saúde, 2006.

DATASUS. Informações em saúde- nascidos vivos. Disponível em http://w3.datasus.gov.br/datasus/datasus.php?area=359A1B378C5D0E0F359G22H0 I1Jd5L25M0N\&VInclude=../site/infsaude.php\&VObj=http://tabnet.datasus.gov.br/cgi/d eftohtm.exe?sinasc/cnv/nv Acesso em 11/01/2008.

GOLDBAUM M. Biblioteca virtual em saúde pública. Rev Saúde Pública Fev;34(1):1-2, 2000.

GOMES, R. , NASCIMENTO, E. F. do. A produção do conhecimento da da saúde pública sobre a relação homem-saúde: uma revisão bibliográfica. Cad. Saúde Pública, Rio de Janeiro, v. 22, n. 5, 2006

GONTIJO, D. T.; MEDEIROS, M. Gravidez/maternidade e adolescentes em situação de risco social e pessoal: algumas considerações. Revista Eletrônica de Enfermagem. v. 06, n. 3, p. 394-399, 2004. Disponível em www.fen.ufg.br.

GONTIJO, D. T.; MEDEIROS, M. "Tava morta e revivi": significado de maternidade para adolescentes com experiência de vida nas ruas. Cadernos de Saúde Pública, vol.24 no.2 Rio de Janeiro Feb. 2008 
HEILBORN, M. L., SALEM, T., ROHDEN, F., BRANDÃO, E., KNAUTH, D., VICTORA, C.; AQUINO, E.; McCALLUM, C.; BOZON, M. Aproximações socioantropológicas sobre a gravidez na adolescência. Horizontes antropológicos, ano 8, n. 17, p. 13-45, 2002

LYRA, J. Paternidade adolescente: da investigação à intervenção. In: ARILHA, M; RIDENTI, S G. U, MEDRADO, B. Homens e Masculinidades: outras palavras. São Paulo: Ecos, , p.185-214.1998

MANCINI, M. C.; SAMPAIO, R. F. Estudo de revisão sistemática: um guia para a síntese criteriosa da evidência científica. Revista Brasileira de Fisioterapia, São Carlos, v. 11, n. 1, p. 83-89, jan./fev. 2007.

\section{REFERÊNCIAS DOS RESUMOS QUE FORAM INCLUÍDOS NA PESQUISA}

ALMEIDA, AM; TRINDADE, RFC; GOMES, FA; NIELSEN, L. Maternidade na adolescência: um desafio a ser enfrentado. Rev. Bras. Enferm.;56(5):519-522, set.-out. 2003

ALMEIDA, MCC; AQUINO, EML; BARROS, AP. School trajectory and teenage pregnancy in three Brazilian state capitals. Cad. Saúde Pública; 22(7):13971409, jul. 2006.

ANDRADE, PR; RIBEIRO, CA; SILVA, CV.O significado do cuidar de seu filho para a mãe adolescente .Acta Paul.. Enferm.;16(4):91-93, out.-dez. 2003.

AQUINO, EML; HEILBORN, ML; KNAUTH, D; BOZON, M; ALMEIDA, MC; ARAÚJO, J; MENEZES, G. Adolescência e reprodução no Brasil: a heterogeneidade dos perfis sociais. Cad. Saúde Pública;19(supl.2):377-388, 2003.

ARAUJO, SG; SANTAANA, DMG. Relação entre a idade materna e o peso ao nascer: um estudo da gravidez na adolescência no município de Umuarama, PR, Brasil em 2001. Ciênc. Cuid. Saúde; 2(2):155-160, jul.-dez.2003.

CAPUTO,V.G; BORDIN, I.A. Gravidez na adolescência e uso freqüente de álcool e drogas no contexto familiar. Rev. Saúde Pública. 42(3):402410,jun,2008.

ARCANJO, CM; OLIVEIRA, MIV; BEZERRA, MGA. Gravidez em adolescentes de uma unidade municipal de Saúde em Fortaleza - Ceará. Esc. Anna Nery Rev. Enferm.;11(3):445-451, set. 2007.

AZEVEDO, DV; SAMPAIO, HAC. Fatores de risco associados à gestação na adolescência. Femina;31(5):457-463, jun. 2003.

AZEVEDO, GE; ABDO, CHN. Adolescentes de classe média do ensino fundamental: prática e conhecimento da sexualidade. Pediatria (São Paulo);28(3):184-190, 2006. i

BARALDI, ACP; DAUD, ZP; Almeida, AM; GOMES, FA; NAKANO, AMS. Adolescent pregnancy: a comparative study between mothers who use public and private health systems. Rev. Latinoam. Enferm.;15(spe):799-805, set.-out. 2007. 
BARROS, DC; PEREIRA, RA; GAMA, SGN; LEAL, MC. O consumo alimentar de gestantes adolescentes no Município do Rio de Janeiro. Cad. Saúde Pública; 20(supl.1):S121-S129, 2004.

BEIRAS, A; TAGLIAMENTO, G; TONELI, MJF. Crenças, valores e visões: trabalhando as dificuldades relacionadas a sexualidade e gênero no contexto escolar.Aletheia;(21):69-78, jan.-jun. 2005.

BELO, MAV; SILVA, JLP. Conhecimento, atitude e prática sobre métodos anticoncepcionais entre adolescentes gestantes. Rev. Saúde Pública; 38(4):479-487, ago. 2004.

BERLOFI, LM; ALKMIN., ELC; BARBIERI, M; GUAZZELLI, CAF; ARAÚJO, FF. Prevenção da reincidência de gravidez em adolescentes: efeitos de um Programa de Planejamento Familiar. Acta Paul.. Enferm.;19(2):196-200, abr.jun. 2006.

BIGRAS, M; PAQUETTE, D. Estudo pessoa-processo-contexto da qualidade das interações entre mãe-adolescente e seu bebê. Ciênc. Saúde coletiva;12(5):1167-1174, set.-out. 2007.

BORGES, ALV; LATORRE, MRDO; SCHOR, N. Fatores associados ao início da vida sexual de adolescentes matriculados em uma unidade de Saúde da família da zona leste do Município de São Paulo, Brasil. Cad. Saúde Pública ;23(7):1583-1594, jul. 2007.

BORGES, ALV; NICHIATA, LYI; SCHOR, N. Conversando sobre sexo: a rede sociofamiliar como base de promoção da Saúde sexual e reprodutiva de adolescentes. Rev. Latinoam. Enferm.;14(3):422-427, maio-jun. 2006.

BORGES, ALV; SCHOR, N. Trajetórias afetivo - amorosas e perfil reprodutivo de mulheres adolescentes residentes no município de São Paulo. Rev. Bras. Saúde matern. infant; 5(2):163-170, abr.-jun. 2005.

BRANDÃO, ER; HEILBORN, ML. Sexualidade e gravidez na adolescência entre jovens de camadas médias do Rio de Janeiro, Brasil. Cad. Saúde Pública ;22(7):1421-1430, jul. 2006.

BRITO, ALS; YAZAKI, LM; MAIA, PB. Vulnerabilidade ao nascer no espaço metropolitano. São Paulo Perspect; 20(1):18-32, jan.-mar. 2006.

CABRAL, CS. Contracepção e gravidez na adolescência na perspectiva de jovens pais de uma comunidade favelada do Rio de Janeiro. Cad. Saúde Pública, 19(supl.2):283-292, 2003.

CAPUTO, VG; BORDIN, IA. Problemas de Saúde mental entre jovens grávidas e não-grávidas. Rev. Saúde Pública ;41(4):573-581, ago. 2007.

CARNIEL, EF; ZANOLLI, ML; ALMEIDA, CAA; MORCILLO, AM. Características das mães adolescentes e de seus recém-nascidos e fatores de risco para a gravidez na adolescência em Campinas, SP, Brasil. Rev. Bras. Saúde Matern.Infant; 6(4):419-426, 2006.

CARVACHO, I.E; PINTO E SILVA, J.L; MELLO, M.B. Conhecimento de adolescentes grávidas sobre autonomia e fisiologia da reprodução. Ver. Ass. Méd. Brasileira. 54(1):29-35, jan-fev, 2008. 
CHALEM, E; MITSUHIRO, SS; FERRI, CP; BARROS, MCM; GUINSBURG, R; LARANJEIRA, R. Gravidez na adolescência: perfil sócio-demográfico e comportamental de uma população da periferia de São Paulo, Brasil. Cad. Saúde Pública ;23(1):177-186, jan. 2007

CONTENTE, TMS; MIRANDA, RNA; GONÇALVES, JFV; LIMA, KCC. Mães adolescentes como fator de risco em recém-nascido de baixo peso: um estudo de meta-análise. Rev. para. med; 19(1):53-57, jan.-mar. 2005.

COSTA, MCO; LIMA, IC; MARTINS JÚNIOR, DF; SANTOS, CAST; ARAÚJO, FPO; ASSIS, DR. Gravidez na adolescência e co-responsabilidade paterna: trajetória sócio-demográfica e atitudes com a gestação e a criança. Ciênc. Saúde coletiva;10(3):719-727, jul.-set. 2005.

COSTA, MCO; SANTOS, CAST; NASCIMENTO SOBRINHO, C; MOURA, MSQ; SOUZA, KEP; ASSIS, DR. Gravidez na adolescência: associação de variáveis sóciodemográficas e biomédicas materna com resultado neonatal. Feira de Santana- Bahia. REV. BAIANA SAÚDE PÚBLICA; 29(2):300-312, jul.-dez. 2005.

COSTA-PAIVA, L; PONTES, LT; HOROVITZ, AP; MARTINS, LBM; FONSECHICARVASAN, GA; Silva, JLP. Perfil social, reprodutivo e sexual de adolescentes atendidas em um ambulatório de ginecologia. Rev. Ciências medicas;13(4):297-305, out.-dez. 2004.

CUNHA, SM; BRUNO, ZV. Reincidência de gravidez na adolescência. Femina;35(11):719-722, nov. 2007.

DALTOSO, D; ALMEIDA, AM; PANOBIANCO, MS. A visão de puérperas adolescentes acerca da atenção pré-natal. Rev. Enferm. UERJ;13(1):83-89, jan.-abr. 2005.

DEL CIAMPO, LA; JUNQUEIRA, MJG; RICCO, RG; DANELUZZI, JC; FERRAZ, IS; MARTINELLI JUNIOR, CE. Tendência secular da gravidez na adolescência. Pediatria (São Paulo); São Paulo, 26(1):21-26, 2003.

DIAS, AB; AQUINO, EML. Maternidade e paternidade na adolescência: algumas constatações em três cidades do Brasil. Cad. Saúde Pública;22(7):1447-1458, jul. 2006.

EDUARDO, KGT; AQUINO, PS; BARBOSA, RCM; PINHEIRO, AKB. Reações da adolescente frente à gravidez. Esc. Anna Nery Rev. Enferm.; 9(2):214-220, ago. 2005.

FALCÃO, DVS; SALOMÃO, NMR. O papel dos avós na maternidade adolescente. Estud. Psicol, Campinas; 22(2):205-212, abr.-jun. 2005.

FERNANDES, JFP; SOUSA, LB; BARROSO, MGT. Repercussão da gravidez no contexto sócio-familiar da adolescente - uma experiência. Acta Paul. Enferm.;17(4):400-406, out.-dez. 2004.

FiORELLI, LR; KREBS, VLJ. Características clínicas e morbidade de recémnascidos filhos de mães adolescentes em hospital universitário. Rev. med. (São Paulo); São Paulo; 85(2):44-49, abr.-jun. 2006.

FLORES SULLCA, T; SCHIRMER, J. Violência intrafamiliar na adolescência na cidade de Puno - Peru.Rev. Latinoam. Enferm.;14(4):579-585, jul.-ago. 2006. 
FOLLE, E; GEIB, LTC. Representações sociais das primíparas adolescentes sobre o cuidado materno ao recém-nascido. Rev. Latinoam. Enferm.;12(2):183-190, mar.-abr. 2004.

FONSECA, AD; GOMES, VLO .Manifestações de gênero no processo de adolescer. Acta Sci, Health Sci;26(1):231-237, jan.-jun. 2004.

FONSECA, ALB; ARAÚJO, NG. Maternidade precoce: uma das consequências do abandono escolar e do desemprego. Rev. Bras. Crescimento desenvolv. hum;14(2):16-22, maio.-ago. 2004.

FONSECA, VS. Percepção de risco: o conceito da adolescente gestante. Rev. Enferm.. UERJ;11(3):278-283, set.-dez. 2003.

GAMA, SGN; SZWARCWALD, CL; SABROZA, AR; CASTELO BRANCO, V; $L E A L, M C$. Fatores associados à assistência pré-natal precária em uma amostra de puérperas adolescentes em maternidades do Município do Rio de Janeiro, 1999-2000. Cad. Saúde Pública; 20(supl.1):S101-S111, 2004.

GOLDANI, MZ; GIUGLIANI, ERJ; SCANLON, T; ROSA, H; CASTILHOS, K; FELDENS, L; TOMKINS, A. Voluntary HIV counseling and testing during prenatal care in Brazil. Rev. Saúde Pública 37(5):552-558, Oct. 2003.

GOLDENBERG, P; FIGUEIREDO, MCT; SILVA, RS. Gravidez na adolescência, pré-natal e resultados perinatais em Montes Claros, Minas Gerais, Brasil. Cad. Saúde Pública; 21(4), jul.-ago. 2005.

GOMIDE, PIC; MILLAN, DC; BOARON, M; RASQUIM, S; CZECZKO, NG; RIBAS, CPM. Práticas parentais educativas e gravidez na adolescência. Rev. med. Paraná; 63(2):32-36, jul.-dez. 2005.

GONÇALVES, H; GIGANTE, D. Trabalho, escolaridade e Saúde reprodutiva: um estudo etno-epidemiológico com jovens mulheres pertencentes a uma coorte de nascimento. Cad. Saúde Pública; 22(7):1459-1469, jul. 2006.

GONTIJO, D.T; MEDEIROS, M. "Tava morta e revivi”: significado de maternidade para adolescentes com experiência de vida nas ruas. Cad. Saúde Pública. 24(2):469-472,fev.2008.

GUERRA, AFFS; HEYDE, MEDVD; MULINARI, RA. Impacto do estado nutricional no peso ao nascer de recém-nascidos de gestantes adolescentes. Rev. Bras. Ginecol. Obstet; 29(3):126-133, mar. 2007.

GURGEL, M. G. I; ALVES, M. D. S; VIEIRA, N. F C; PINHEIRO, P. N. C; BARROSO, G.T. Gravidez na adolescência: tendências na produção científica de enfermagem: revisão. Anna Nery; 12(4):799-805, dez.2008.

HOGA, L.A.K. Maternidade na adolescência em uma comunidade de baixa renda:experiências reveladas pela história oral. Rev Latino-am Enfermagem. 16(2), março-abril. 2008.

HORTA, BL; VICTORA, CG; GIGANTE, DP; SANTOS, J; BARROS, FC.Duração da amamentação em duas gerações. Rev. Saúde Pública ;41(1):13-18, fev. 2007.

JORGE, MSB; FIÚZA, GV; QUEIROZ, MVO. Existential phenomenology as a possibility to understand pregnancy experiences in teenagers. Rev. 
Latinoam. Enferm.;14(6):907-914, nov.-dez. 2006.

KAC, G; VELÁSQUEZ-MELÉNDEZ, G; VALENTE, JG Menarca, gravidez precoce e obesidade em mulheres brasileiras selecionadas em um Centro de Saúde de Belo Horizonte, Minas Gerais, Brasil. Cad. Saúde Pública, 19(supl.1):S111-S118, 2003

KASSAR, SB; GURGEL, RQ; ALBUQUERQUE, MFM; BARBIERI, MA; LIMA, MC. Peso ao nascer de recém-naScidos de mães adolescentes comparados com o de puérperas adultas jovens. Rev. Bras. Saúde matern. infant;5(3):293-299, jul.-set. 2005.

KASSAR, SB; LIMA, MC; ALBUQUERQUE, MFM; BARBIERI, MA; GURGEL, RQ. Comparações das condições socioeconômicas e reprodutivas entre mães adolescentes e adultas jovens em três maternidades Públicas de Maceió, Brasil. Rev. Bras. Saúde Matern. Infant;6(4):397-403, 2006.

LEAL, AC; WALL, ML. Percepções da gravidez para adolescentes e perspectivas de vida diante da realidade vivenciada. Cogitare Enferm.;10(3):44-52, set.-dez. 2005.

LEVANDOWKI, D.C; PICCININI, C.A; LOPES, R.C.S. Maternidade adolescente. Est. Psicologia; 25 (2):251-263, abr-jun, 2008.

LOSS, MA; SAPIRO, CM. Processos psíquicos do engravidamento na adolescência em contextos de periferia: impasses e possibilidades. Psicol. USP; São Paulo, 16(4):69-98, 2005.

MAGALHÃES, MLC; FURTADO, FM; NOGUEIRA, MB; CARVALHO, FHC; ALMEIDA, FML; MATTAR, R; CAMANO, L. Gestação na adolescência precoce e tardia: há diferença nos riscos obstétricos? Rev. Bras. Ginecol. Obstet; 28(8):446-452, ago. 2006

MAIA, FFR; ANDRADE, CG; MAAKAROUN, MF. Anticoncepção na primeira relação sexual como fator de risco para a gravidez em adolescentes. Rev Med. Minas Gerais;13(1):4-8, jan.-mar. 2003.

MAIA, VOA; MAIA, ACA; QUEIROGA, FL; MAIA FILHO, VOA; ARAÚJO, AB; LIPPO, LAM; ALBUQUERQUE, RM. Via de parto em gestações sucessivas em adolescentes: estudo de 714 casos. Rev. Bras. Ginecol. Obstet;26(9):703707, out. 2004.

MARQUES, R.F.S.V; CUNHA, I.C.C; ARAGON, M.G; PEIXOTE, V.S. Fatores relacionados às dificuldades no aleitamento materno entre mães adolescentes da Fundação Santa Casa de Misericórdia do Pará. Rev. Paraense.Med. 22 (1):5762, Jan-Mar, 2008.

MARTINEZ, LDC; FERRIANI, MGC. Relación entre las características de la adolescente embarazada y la resistencia al consumo de droga. Rev. Latinoam. Enferm.;12(n.esp):333-339, mar.-abr. 2004.

MATURANA, HCA; PROGIANTI, JM. A ordem social inscrita nos corpos: gravidez na adolescência na ótica do cuidar em Enfermagem. Rev. Enferm. UERJ;15(2):205-209, abr.-jun. 2007. 
MAUCH, SDN; CABRAL, CMC; PINHEIRO, ZED; PARCA, JM. Gravidez na adolescência: um estudo sobre o problema em Santa Maria-DF. Brasilia med; 42(1/2):16-23, 2005.

MENEZES, GMS; AQUINO, EML; SILVA, DO. Induced abortion during youth: social inequalities in the outcome of the first pregnancy. Cad. Saúde Pública ;22(7):1431-1446, jul. 2006.

MENEZES, IHCF; DOMIN.GUES, MHMS. Principais mudanças corporais percebidas por gestantes adolescentes assistidas em serviços públicos de Saúde de Goiânia. Rev. nutr;17(2):185-194, abr.-jun. 2004.

MITSUHIRO, SS; CHALEM, E; BARROS, MM; GUINSBURG, R; LARANJEIRA, R .Teenage pregnancy: use of drugs in the third trimester and prevalence of psychiatric disorders. Rev. Bras. psiquiatr; 28(2):122-125, jun. 2006.

MONTEIRO, CFS; COSTA, NSS; NASCIMENTO, PSV; AGUIAR, YA. A violência intra-familiar contra adolescentes grávidas. Rev. Bras. Enferm.;60(4):373-376, jul.-ago. 2007.

MORAES, AJP; SOARES, PMF; LEAL, MM; SALLUM, AME; LOTITO, APN; SILVA, CAA. Aspectos da gravidez e pós-parto de adolescentes portadoras de febre reumática. Rev. Assoc. Med. Bras. ;50(3):293-296, jul.-set. 2004.

MOREIRA, MC; SARRIERA, JC. Preditores de Saúde e bem-estar Psicológico em adolescentes gestantes. Psico USF;11(1):7-15, jan.-jun. 2006.

MOREIRA, T.M.M; VIANA, D.S; QUEIROZ, M.V.O; JORGE, M.S.B. Conflitos vivenciados pelas adolescentes com a descoberta da gravidez. Rev.Es.Enf.USP, São Paulo. 42(2):312-320, jun.2008.

MORESCO, JO; VAN DER SAND, ICP. Das bonecas ao bebê: a vida da adolescente ao tornar-se mãe. Sci med;15(1):34-41, 2005.

MOTTA, MGC; RIBEIRO, NRR; PEDRO, ENR; COELHO, DF. Vivências da mãe adolescente e sua família. Acta Sci, Health Sci; 26(1):249-256, jan.-jun. 2004.

NOGUEIRA, AM; MARCON, SS. Reações, atitudes e sentimentos de pais frente a gravidez na adolescência. Ciênc. Cuid. Saúde;3(1):23-32, jan.-abr. 2004.

NOGUEIRA, NN; PARENTE, JV; COZZOLINO, SMF. Mudanças na concentração plasmática de zinco e ácido fólico em adolescentes grávidas submetidas a diferentes esquemas de suplementação. Cad. Saúde Pública, 19(1):155-160, jan.-fev. 2003.

NUNES, SOV; ABREU, RE; HIRATA, AL; NUNES, MVA; FRANCO, RM; BARBOSA, LR. Determinação dos diagnósticos de depressão, tentativa de suicídio, gravidez, vírus da imunodeficiência humana (HIV) e doenças sexualmente transmissíveis (DST) em adolescentes e adultos jovens. Semina Ciênc. biol. Saúde; 26(2):109-118, out.-dez. 2005.

NUNESMAIA, HGS; MACHADO, MA; NÓBREGA, FPM. Intencionalidade Reprodutiva em Universitários de João Pessoa - Paraíba - Brasil. Rev. Bras. Ciênc. Saúde; 7(2):155-160, 2003. 
OLIVEIRA, NR. Maternidade de adolescentes de periferias sociais e urbanas: algumas análises à luz da Psicologia ambiental. Rev. Bras. Crescimento desenvolv. hum;15(1):69-77, jan.-abr. 2005.

ORLANDI, R; TONELI, MJF. Sobre o processo de constituição do sujeito face à paternidade na adolescência. Psicol. Rev. (Belo Horizonte);11(18):257-267, dez. 2005.

OTSUKA, F; NARAHARA, J; AYABE, L; CACCELLI, M; SALINA, V; MOLINOS, V; MARTINS, LC; LUIZ, OC. O programa de Saúde da família e a gravidez na adolescência em São Bernardo do Campo. Arq. med. ABC; 30(2):90-93, 2005.

PAIVA, SS; GALVÃO, MTG. Gravidez em adolescente com infecção pelo HIV/AIDS. Rev. Enferm.UERJ; 14(4):586-591, out.-dez. 2006 .

PANTOJA, ALN. Ser alguém na vida: uma análise sócio-antropológica da gravidez/maternidade na adolescência, em Belém do Pará, Brasil. Cad. Saúde Pública. 19(supl.2): 335-343, 2003.

PAPA, ACE; FURLAN, JP; PASQUELLE, M; GUAZZELLI, CAF; FIGUEIREDO, MS; CAMANO, L; MATTAR,R. A anemia por deficiência de ferro na grávida adolescente: comparação entre métodos laboratoriais. Rev. Bras. Ginecol. Obstet; 25(10):731-738, nov.-dez. 2003.

PARAGUASSÚ, ALCB; COSTA, MCO; NASCIMENTO SOBRINHO, CL; PATEL, BN; FREITAS, JT; ARAÚJO, FP O. Situação sociodemográfica e de Saúde reprodutiva pré e pós-gestacional de adolescentes, Feira de Santana, Bahia, Brasil. Ciênc. Saúde coletiva; 10(2):373-380, abr.-jun. 2005.

PEDRO, ENR; BOTENE, DZA; MOTTA, MGC; RIBEIRO, NRR; LIMA, AAA. O desenvolvimento do apego da mãe adolescente e seu bebê. Online Braz. J.Nurs. (Online);6(2), ago. 2007.

PERES, SO; HEILBORN, ML. Cogitação e prática do aborto entre jovens em contexto de interdição legal: o avesso da gravidez na adolescência. Cad. Saúde Pública; 22(7):1411-1420, jul. 2006.

PERSONA, L; SHIMO, AKK; TARALLO, MC. Perfil de adolescentes com repetição da gravidez atendidas num ambulatório de pré-natal. Rev. Latinoam. Enferm.;12(5):745-750, set.-out. 2004.

PICCININI, CA; FERRARI, AG; LEVANDOWSKI, DC; LOPES, RS; NARDI, TC. 0 bebê imaginário e as expectativas quanto ao futuro do filho em gestantes adolescentes e adultas. Interações Estud. Pesqui. Psicol; 8(16):81-108, jul.-dez. 2003.

PINHO, LB; MEINCKE, SMK. Conhecendo a clientela do pré-natal de um serviço da rede Pública. Rev. Enferm. UERJ;11(1):52-58, 2003.

PINTO, LF; MALAFAIA, MF; BORGES, JA; BACCARO, A; SORANZ, DR. Perfil social das gestantes em unidades de Saúde da família do município de Teresópolis. Ciênc. Saúde coletiva; 10(1):205-213, jan.-mar. 2005.

PIROTTA, KCM; SCHOR, N. Intenções reprodutivas e práticas de regulação da fecundidade entre universitários. Rev. Saúde Pública; 38(4):495-502, ago. 2004 
POLI, ME; MACHADO, J. Um caso de gestação na adolescência: algumas considerações práticas e bioéticas. Sci med; 15(1):42-46, 2005.

PUHL, CH; PEREIRA, LDC; GRISARD, N; HALLAL, ALC. Morbimortalidade do recém-nascido de mãe adolescente. ACM; 36(3):52-58, 2007.

RANGEL, D.L.O; QUEIROZ, A.B.A. A representação social ds adolescentes sobre a gravidez nesta etapa da vida. Rev. Anna Nery; 12(4):780788,dez.2008.

REIS, AOA; OLIVEIRA-MONTEIRO, NR. Sexualidade e procriação na ótica de jovens de periferias sociais e urbanas. Rev. Bras. Crescimento Desenvolv.Hum;17(2):54-63, abr.-jun. 2007.

REIS, E; CAVALCANTI, SMOC. O significado da gravidez para a adolescente. Rev. Saúde Dist. Fed;16(1/2):27-34, jan.-jun. 2005.

RIBEIRO, ACL; UHLIG, RFS .A gestação na adolescência e a importância da atenção a Saúde do adolescente. Divulg. Saúde Debate; (26):30-36, abr. 2003.

ROCHA, DCS; BEZERRA, MGA; CAMPOS, ACS. Cuidados com os bebês: o conhecimento das primíparas adolescentes. Esc. Anna Nery Rev. Enferm.;9(3):365-371, dez. 2005.

RODRIGUES, EAS; SOUZA, EP; GUEDES, CC; MADEIRA, AMF. 0 adolescente e a vivência da paternidade: uma abordagem fenomenológica. REME Rev. Min. Enferm.;7(2):82-88, jul.-dez. 2003.

RODRIGUES, F.R.A; RODRIGUES, D.P;SOUZA, E.S; NOGUEIRA, M.E.F; FIALHO, A.V.M. A vivência do ciclo gravídico-puerperal na adolescência: perfil sociodemográfico e obstétrico. Rev. Min.Enfer. 12(1):27-33, janmar.2008.

ROMERO, KT; MEDEIROS, ÉHGR; VITALLE, MSS; WEHBA, J.O conhecimento das adolescentes sobre questões relacionadas ao sexo. Rev. Assoc. Med. Bras. ;53(1):14-19, jan.-fev. 2007.

ROSA, AJ; REIS, AOA; TANAKA, ACA. Gestações sucessivas na adolescência. Rev. Bras. Crescimento Desenvolv.Hum;17(1):165-172, jan.-mar. 2007.

SABROZA, AR; LEAL, MC; GAMA, SGN; COSTA, JV. Perfil sócio-demográfico e psicossocial de puérperas adolescentes do Município do Rio de Janeiro, Brasil - 1999-2001. Cad. Saúde Pública; 20(supl.1):S112-S120, 2004.

SABROZA, AR; LEAL, MC; SOUZA JÚNIOR, PR; GAMA, SGN. Algumas repercussões emocionais negativas da gravidez precoce em adolescentes do Município do Rio de Janeiro (1999-2001). Cad. Saúde Pública , 20(supl.1):S130-S137, 2004.

SAITO, MI; LEAL, MM. O exercício da sexualidade na adolescência: a contracepção em questão. Pediatria (São Paulo);25(1/2):35-42, 2003.

SANTOS, A; CARVALHO, CV. Gravidez na adolescência: um estudo exploratório. Bol. Psicol; 56(125):135-151, jul.-dez. 2006.

SANTOS, SR; SCHOR, N. Vivências da maternidade na adolescência precoce. Rev. Saúde Pública. 37(1):15-23, fev. 2003. 
SARAIVA, KVO; COSTA, L; XIMENES, LB. Prática de Enfermagem com terapias alternativas em adolescentes grávidas. Texto \& contexto Enferm.;12(2):151-157, abr.-jun. 2003.

SILVA, CAA; LEAL, MM; FEBRÔNIO, MV; LEAL, GN; SALLUM, AME; ANDRADE, JQ; ZUGAIB, M. Gravidez em adolescentes com dermatomiosite juvenil ( DMJ ). Rev. Bras. Reumatol; 45(3):180-184, maio-jun. 2005.

SILVA, DR.S; INAGAKI, A.D.M; DALTRO, A.S.T. Intercorrências clínicas e obstétricas da gravidez na adolescência. Nursing; 11 (123):382-386,ago,2008.

SILVA, L; TONETE, VLP. A gravidez na adolescência sob a perspectiva dos familiares: compartilhando projetos de vida e cuidado. Rev. Latinoam. Enferm.;14(2):199-206, mar.-abr. 2006.

SILVEIRA, IP; OLIVEIRA, MIV; FERNANDES, AFC. Perfil obstétrico de adolescentes de uma maternidade Pública no Ceará. Esc. Anna Nery Rev. Enferm.;8(2):205-210, ago. 2004.

SIMÖES, VMF; SILVA, AAM; BETTIOL, H; LAMY-FILHO, F; TONIAL, SR; MOCHEL, EG. Características da gravidez na adolescência em São Luís, Maranhão. Rev. Saúde Pública, 37(5):559-565, out. 2003.

SOARES, S. M; AMARAL, M. A; SILVA, L.B; SILVA, P.A.B. Oficinas sobre sexualidade na adolescência: revelando vozes, desvelando olhares de estudantes do ensino médio. Esc. Anna Nery Enf. 12 (3): 485-491, set. 2008.

TAVARES, BB; FERRARI, DC; SOLER, ZASG. Caracterização da gestação e parto das adolescentes de São José do Rio Preto em 2003. Arq. Ciênc. Saúde;13(1):12-17, jan.-mar. 2006.

TROTTA, G; NOVO, NF; JULIANO, Y; SIGULEM, DM; MANTESE, JC; BARROS, ACSD; CURY, MCFS; CAVAGNA, M. Gravidez na adolescência: resultados obstétricos e neonatais. Reprod. clim;22:35-40, 2007.

VIEIRA, LM; GOLDBERG, TBL; SAES, SO; DÓRIA, AAB. Abortamento na adolescência: um estudo epidemiológico. Ciênc. Saúde coletiva; 12(5):12011208, set.-out. 2007.

VIEIRA, LM; SAES, SO; DÓRIA, AAB; GOLDBERG, TBL. Reflexões sobre a anticoncepção na adolescência no Brasil. Rev. Bras. Saúde Matern.Infant, 6(1):135-140, jan.-mar. 2006.

VILLELA, WV; DORETO, DT. Sobre a experiência sexual dos jovens. Cad. Saúde Pública; 22(11):2467-2472, nov. 2006.

VITALLE, MSS; AMANCIO, OMS. Tabagismo e gravidez. Rev. Paul. Pediatr ;21(1):27-32, mar. 2003.

XIMENES NETO, FRG; DIAS, MSA; ROCHA, J; CUNHA, ICKO. Gravidez na adolescência: motivos e percepções de adolescentes. Rev. Bras. Enferm.; 60(3):279-285, maio-jun. 2007. 